\title{
Have Severity Scores a Place in Predicting Septic Complications in ICU Multiple Trauma Patients?
}

\author{
Daniela lonescu \\ Department of Anesthesia and Intensive Care I, "Iuliu Hatieganu” University of Medicine and Pharmacy, Cluj-Napoca, \\ Romania; Outcome Research Consortium, Cleveland, USA
}

Risk assessment in ICU critically-ill patients is of tremendous importance for optimizing patients' clinical management, medical and human resource allocation and supporting medical cost distribution and containment.

The problem of predicting complications and mortality in ICU patients, although not new, is of genuine concern and much effort has been made to detect the most reliable parameters and scores. Numerous attempts have been made to use clinical and laboratory findings integrated into different algorithms or to incorporate these parameters into easy to use composite severity scores which would be applicable in various centers. In addition to clinical data, biomarkers or laboratory findings have been used for this purpose [1-3].

The SOFA, SAPS and APACHE scores and their newer versions, have been used worldwide to evaluate patients' severity, prognosis, and survival [4-7]. However, it has been reported that there are differences in their performance and estimation probability, in different geographical areas [8].

The applicability of composite scores to various categories of ICU patients, e.g. septic, trauma, cancer, and others, is also a matter of debate and is the subject of several studies $[9,10]$.

In trauma patients, there have been numerous attempts to use "general" or specific scores and to compare the results $[6,7,11]$ and scores like ISS and RTS have been developed and used to estimate survival and risks in trauma patients [4-7].

On the other hand, sepsis is the most important cause of late mortality in severe trauma patients, $[4,12,13]$ and attempts have been made to predict risk of septic complications in these patients.
Tranca et al. reported a study which aimed at identifying the most accurate method or score to predict the risk of septic complications in multiple trauma patients [14]. The study also investigated if severity scores may be used for this purpose and if there were highly predictive cut-off values for septic complications in multiple trauma patients. The authors found that both functional severity and specific scores might be used to predict risks of septic complications in multiple trauma patients and describe cut-off values for these scores which are most reliable in predicting infectious complications in this category of patients. Thus a SOFA score $>4$ and an APACHE II $\geq 11$ were predictive of developing septic complications, as were the trauma scores, RTS $\geq 7$ and ISS $\geq 22$, in predicting infectious complications in multiple trauma patients. The data was collected from a single center, and the population sample size was relatively small, both factors setting limits on the robustness of the research. Similar results have been reported in others studies [15-17], however Singh et al. reported different findings. He used trauma and injury severity score (TRISS), a combination index based on revised trauma score (RTS), injury severity score (ISS) and patient's age to predict the outcome in trauma cases [18] and the performance of TS, ISS and TRISS as predictors of survival was evaluated using the misclassification rate, the information gain and the relative information gain. A graded increase in mortality with decreasing RTS score was reported. It would have been interesting if Tranca et al would have comment in their article more on their results and especially on cutoff values in relation with the literature.

In conclusion, further studies are needed to find the best scores and reference values that predict the risk of septic complications in ICU multiple trauma patients. 
108 The Journal of Critical Care Medicine 2016;2(3)

Inter-center as well as inter-patient category studies would lead to a better clinical validation of these scores, as well as giving rise to better predictions of outcome measures. Concomitantly this would lead to better clinical management and resources allocation.

\section{REFERENCES}

1. Kumar A, Roberts D, Wood KE, et al. Duration of hypotension before initiation of effective antimicrobial therapy is the critical determinant of survival in human septic shock. Crit Care Med. 2006;34:1589-96.

2. Kibe S, Adams K, Barlow G. Diagnostic and prognostic biomarkers of sepsis in critical care. J. Antimicrob Chemother. 2011;66:33-40.

3. Tanriverdi H, Tor MM, Kart L, Altnn R, Atalay F, Sumb Sümbüloğlu V. Prognostic value of serum procalcitonin and C-reactive protein levels in critically ill patients who developed ventilator-associated pneumonia. Annals of Thoracic Medicine. 2015;10:137-42.

4. Antonelli M, Moreno R, Vincent JL, Sprung CL, Mendoça A, Passariello $\mathrm{M}$, et al. Application of SOFA score to trauma patients. Sequential Organ Failure Assessment. Intensive Care Med. 1999;25:389-94.

5. Mica L, Furrer E, Keel M, Trentz O. Predictive ability of the ISS, NISS, and APACHE II score for SIRS and sepsis in polytrauma patients. Eur J Trauma Emerg Surg. 2012;38:665-71

6. Agarwal A, Agrawal A, Maheshwari R. Evaluation of Probability of Survival using APACHE II \& TRISS Method in Orthopaedic Polytrauma Patients in a Tertiary Care Centre. Journal of Clinical and Diagnostic Research. JCDR. 2015;9:RC01-RC04.

7. Brattström O, Granath F, Rossi P, Oldner A. Early predictors of morbidity and mortality in trauma patients treated in the intensive care unit. Acta Anaesthesiol Scand. 2010;54:1007-17.

8. Katsounas A, Kamacharoval, Tyczynski B, et al. The predictive performance of the SAPS II and SAPS 3 scoring systems: A
Available online at: www.jccm.ro

retrospective analysis. J Crit Care. 2016;33:180-5.

9. Soares M, Salluh JI. Validation of the SAPS 3 admission prognostic model in patients with cancer in need of intensive care. Intensive Care Med. 2006;32: 1839-44.

10. Schellongowski $P$, Schellongowski $P$, Benesch $M$, et al. Comparison of three severity scores for crtically ill cancer patients. Intensive Care Med. 2004;30:430-6.

11. Keel M, Eid K, Labler L, Seifert B, Trentz O, Ertel W. Influence of injury pattern on incidence and severity of posttraumatic inflammatory complications in severely injured patients. Eur J Trauma. 2006;32:387-95.

12. Pfeifer R, Tarkin IS, Rocos B, Pape HC. Patterns of mortality and causes of death in polytrauma patients--has anything changed? Injury. 2009;40:907-11.

13. Jin H, Liu Z, Xiao Y, Fan X, Yan J, Liang H. Prediction of sepsis in trauma patients. Burn Trauma. 2014;2:106-13.

14. Tranca S, Petrișor C, Hagău N, Ciuce C. Can severity scores APACHE II, SOFA, ISS, and RTS be used to predict ICU septic complications in multiple trauma patients? J Crit Care Med. 2016;3:124-30.

15. Wafaisade A, Lefering R, Bouillon B, Sakka SG, Thamm OC, Paffrath $T$, et al.; Trauma Registry of the German Society for Trauma Surgery. Epidemiology and risk factors of sepsis after multiple trauma: An analysis of 29,829 patients from the Trauma Registry of the German Society for Trauma Surgery. Crit Care Med. 2011;39:621-8.

16. Osborn TM, Tracy JK, Dunne JR, Pasquale M, Napolitano LM. Epidemiology of sepsis in patients with traumatic injury. Crit Care Med. 2004;32:2234-40.

17. Kisat M, Villegas CV, Onguti S, Zafar SN, Latif A, Efron DT, et al. Predictors of sepsis in moderately severely injured patients: An analysis of the National Trauma Data Bank. Surg Infect (Larchmt). 2013;14:62-8.

18. Singh J, Gupta G, Garg R, Gupta A. Evaluation of trauma and prediction of outcome using TRISS method. Journal of Emergencies, Trauma and Shock. 2011;4:446-9. 